\title{
PREDICTORS OF PSYCHOLOGICAL MORBIDITY IN PARENTS OF CHILDREN WITH INTELLECTUAL DISABILITY
}

\author{
DR. PRITAM CHANDAK \\ MBBS M.D (Psychiatry) \\ M.APA (USA) M.EPA (EUROPE) M. ACAMH (UK) M.IPS \\ Fellowship (Child \& Adolescent Psychiatry), London \\ Diploma (Child play therapy), London \\ Clinical fellow (Child \& Adolescent Psychiatry), NIMHANS
}

Objective: This study investigated symptoms of depression and anxiety and their origins in parents caring for intellectually disabled children relative to parents of typically developing children.

Design: A cross-sectional case control study.

Methods: Thirty two parents of children with intellectual disabilities and twenty nine parents of typically developing children completed measures of anxiety and depression, social support, child problem behaviours, sleep quality and caregiver burden.

Results: The parents of children with intellectual disabilities registered much higher depression and anxiety scores on the Hospital Anxiety and Depression Scale and the majority met the established criteria for possible clinical depression and/or anxiety. Of the putative mediators measured, the strongest and most consistent predictor of group differences in distress, as well as individual variations in distress within the group of parents with intellectually disabled children, was caregiver burden. Analyses of its component dimensions indicated that the negative psychosocial consequences of caregiving were particularly predictive of depression, whereas guilt was the main associate of anxiety.

Conclusions: Symptoms of depression and anxiety are highly prevalent in parents of children with intellectual disabilities and different dimensions of caregiver burden predicted depression and anxiety. This suggests that, in developing interventions for this population, attention should be paid to the way in which parental distress presents itself as depression, anxiety, or both, in the individual caregiver.
Table 1. Demographic characteristics and child care responsibilities of parental groups

\begin{tabular}{lccl}
\hline & Cases (N $=32)$ & Controls (N $=29)$ & Test of difference \\
\hline Sex (Female) & $24(75 \%)$ & $20(69 \%)$ & $\chi^{2}(1)=0.06, p=.81$ \\
Marital Status (Partnered) & $28(88 \%)$ & $20(70 \%)$ & $\chi^{2}(1)=2.11, p=.15$ \\
Ethricity (Caucasian) & $29(91 \%)$ & $26(90 \%)$ & $\chi^{2}(1)=0.00, p=1.00$ \\
Occupational status (Professional) & $15(47 \%)$ & $16(55 \%)$ & $\chi^{2}(1)=0.15, p=.70$ \\
Currently employed outside the home & $22(69 \%)$ & $29(100 \%)$ & $\chi^{2}(1)=7.19, p=.007$ \\
Mean age (SD) years & $42.8(5.78)$ & $39.9(4.36)$ & $\mathrm{F}(1,59)=4.76, p=.03$ \\
Mean body mass index (SD) $\mathrm{kg} / \mathrm{m}^{2}$ & $26.8(4.27)$ & $24.7(4.10)$ & $\mathrm{F}(1,56)=3.80, p=.06$ \\
Primary caregiver & $25(78 \%)$ & $21(72 \%)$ & $\chi^{2}(1)=0.05, p=.83$ \\
Mean age of main care recipient (SD) years & $11.2(3.99)$ & $8.9(4.57)$ & $\mathrm{F}(1,54)=3.88, p=.06$ \\
\hline
\end{tabular}

Table 2. Symptoms of depression and anxiety by parent group

\begin{tabular}{lll}
\hline & Cases (N=32) & Control (N=29) \\
\hline Mean depression score (SD) & $8.6(3.92)$ & $3.2(2.31)$ \\
Mean anxiety score (SD) & $11.0(4.40)$ & $5.0(2.44)$ \\
Depression score $\geq 8(\%)$ & $20(63 \%)$ & $2(7 \%)$ \\
Anxiety score $\geq 8(\%)$ & $24(75 \%)$ & $3(10 \%)$ \\
\hline
\end{tabular}

Table 3. Social support, sleep quality, child problem behaviours, caregiving burden by parent group

\begin{tabular}{lcc}
\hline & & \\
& Cases $(\mathrm{N}=32)$ & Control $(\mathrm{N}=29)$ \\
\hline Mean social support score (SD) & $31.3(9.76)$ & $37.9(10.37)$ \\
Mean sleep quality score (SD) & $10.0(2.48)$ & $7.1(2.09)$ \\
Mean child behaviour problem score (SD) & $22.9(5.88)$ & $9.9(4.89)$ \\
Mean caregiver burden score (SD) & $44.0(14.26)$ & $22.9(10.67)$
\end{tabular}

